\title{
The Cosmos with the Success/failure System
}

\author{
Dong-Yih Bau
}

\author{
Department of Information Management, Da-Yeh University,168 University Rd., Dacun, \\ Changhua 51591, Taiwan, R.O.C.
}

\begin{abstract}
The ancient Greeks saw the universe as a wellordered system called the cosmos. Current scientific research seeks the cosmos in terms of a final theory, which remains in progress. By applying Einstein's principle theory, we developed the success/failure system hypothesis (success/failure system) and recently made it available in the literature. Herein, we consider the cosmos subsequent to the existence of this hypothesis. After a brief introduction, we focus on two themes: the success/failure system principle and a final theory of our universe. A key message is that the success/failure system principle is a new universal biological principle identified in the twenty-first century, and the success/failure system hypothesis may need to be incorporated into a final theory of the life-laden universe.
\end{abstract}

Keywords: Biological law, Einstein, Final theory, Principle theory, Success/failure system, The cosmos

\section{Introduction}

Whether there are universal laws in biology is a longstanding issue ${ }^{1-3}$. Although Einstein remains prominent in popular literature for his quirks and delightful aphorisms, his scientific thought, which included principle theory, touched upon this question of universal laws ${ }^{4-6}$. Remarkably, he expressed the cosmic analysis that he wished to experience as complex but not totally chaotic, with the universe as a single significant whole and thus, an orderly $\operatorname{cosmos}^{4,6}$. In this regard, Einstein is a special mind in humanity. Recently, we used Einstein's principle theory to identify a new universal biological principle called the success/failure system principle and develop the success/failure system hypothesis ${ }^{7}$. This paper is based on the hypothesis, and focuses on its implications regarding the cosmos after success/failure system. The reader is requested to examine the literature regarding this hypothesis ${ }^{7}$ before studying this short paper.

\section{The Success/failure System Principle}

Principle theory ${ }^{4,6}$ is a constraining method for cosmic analysis, which resulted from Einstein's philosophical and methodological reflections on his scientific achievements, including general relativity. He had a conviction that the universe has a priori harmony, and thus believed that the universe is a perfect structure by mathematical reasoning and logical analysis ${ }^{4,6}$. By considering the universe as a significant whole while building up the cosmos with a minimum of primary concepts and relations, Einstein aimed to develop a highly constrained theoretical analysis of the cosmos ${ }^{4,6}$. This aim seemed unattainable, but a fine example is general relativity, which provides the choreography for an entwined cosmic dance of space, time, matter, and energy among celestial bodies in the universe $e^{4,5}$.

The theorist's method $^{4,6}$ involves first identifying general postulates or principles from which he can then deduce conclusions. There is no standard method of establishing principles. The theorist must develop or deduce general principles out of nature by intuitively grasping the essentials of a large complex set of facts that permit precise formulation in axioms ${ }^{4}$. This demands the highest possible standard of accurate and logical description of the cosmos that mathematics can give ${ }^{4}$. In short, principle theory is a 'scientific' axiomatic system, just as Euclidean geometry is an axiomatic system in mathematics, and thus has the properties of supreme purity, clarity, and certainty ${ }^{4}$. However, principles are rare and hard to find, as experienced by Einstein ${ }^{6}$. Furthermore, a scientific principle theory is the result of the unique discovery process of mathematical reasoning and logical analysis rather than simply the output of the general descriptive method ${ }^{4}$.

Whereas many knew Einstein's scientific interest in the structure of the universe, few knew his interest in the eternity of life, which he mentioned in passing in the literature ${ }^{4,6}$. For example, death is a reality ${ }^{6}$; scientific research assumes that all events, including the actions of mankind, are determined by the laws of nature ${ }^{4,6}$. We took the opportunity of exploring the eternity of life by using Einstein's principle theory to identify the success/failure system principle and develop the success/failure system hypothesis ${ }^{7}$. Our hypothesis uses the discrete mathematics of partial ordering (PO) to understand the success/failure system ${ }^{7}$. The mathematical formulation of the success/failure system principle is $\mathrm{PO}$ conditions for success $=\mathrm{PO}$ causes of failure, which indicates the same partial ordering for two distinct relations? ${ }^{7}$ We also 
articulated this success/failure system principle by referring to its logical part-whole relationship and the associated hierarchical structure, as well as the dependency relations of the conditions for success and the causes of failure between any two consecutive levels in the hierarchical structure ${ }^{7}$.

The success/failure system principle is a symmetry principle of all systems, which is one among all internal symmetries of laws within our universe. Success/failure systems include the panorama of life and of human contrivances ${ }^{7}$. Indeed, within our own discipline, even business organizations and ongoing innovations in information technology follow (or accord with) the success/failure system principle. The success/failure system hypothesis focuses on life, and implies that every planet has the potential to evolve into a success/failure system ${ }^{7}$. Drawing this conclusion was simply mathematical reasoning based on the number of levels in the system ${ }^{7}$. In our experience applying principle theory, we have also seen just one unique solution for the eternity of life, which coincides with the structure of the universe as experienced by Einstein ${ }^{4}$. The success/failure system hypothesis provides the choreography for a different but similarly entwined cosmic dance of success, failure, part, and whole in all systems of life and (human) contrivances?

Thus, the success/failure system principle is a new universal biological principle defined in the twentyfirst century ${ }^{7}$. Einstein's principle theory ${ }^{4,6}$ provided the method to discover this principle. Einstein claimed that using the inductive method to identify a universal principle would fail ${ }^{4}$, as in biological mathematical theory ${ }^{8}$. Thus, what does this new universal principle signify in biology? We argue further that this principle is the only universal law (or principle) in biology.

Although the success/failure system principle, as a principle theory, is a logical system, it can serve as a theoretical framework for explaining the historic practice and divisions of biology. Living organisms form a hierarchy of ever more complex systems ${ }^{2,3,9}$. Botany, entomology, ornithology, zoology, and other disciplines, which centre on groups of organisms and try to know everything about the organic systems, reflect the old division of biology $y^{2,3,9}$. However, with the vast experience and literature now available, biological analysis can be thought of differently ${ }^{2,3,9}$. Thus, biology is now sliced crosswise, according to levels of biological organization, oriented toward the molecule, cell, organism, population, and ecosystem $^{2,3,9}$. The current division of biology into molecular and cell biology, evolutionary biology, systems biology, ecology and so forth aims to overcome the practical limit to complexity and simultaneously grasp empirical laws and regularities to suggest adaptive hypotheses from new data ${ }^{2,3,9}$. In this regard, for example, DNA forms the basis of molecular and cell biology, a major contribution to the unification of biology, while Darwin's theory of evolution serves as a theoretical framework for evolutionary biology. However, both DNA and Darwin's theory of evolution are applicable in only some aspects of biology, in contrast to the universal biological principle, the success/failure system principle. To use Einstein's term ${ }^{4}$, the success/failure system principle forms the theoretical foundation of biology. The success/failure system hypothesis even suggests the existence of theories about the potentialities of life in the universe proposed in the literature ${ }^{10}$, which astrobiology focuses on currently. Note that the success/failure system principle can account for every division of biology ever practiced on Earth, or elsewhere.

\section{A Final Theory of Our Universe}

Since the second half of the twentieth century, many physicists and mathematicians have been vigorously conducting a great intellectual adventure to determine how the universe operates via the search for a final theory ${ }^{11-17}$ : Scientists seek the fundamental principles (or laws) from which all other scientific principles can be derived. This would frame the universe as a well-ordered system; this was called the cosmos by the ancient Greeks, who were able to intuit but not express the concept of a unified theory. Scientists have achieved a consensus about what laws of nature must be incorporated into a final theory ${ }^{11-14}$. First, quantum mechanics is needed to address the microscopic structure of the universe, the microcosmos. Second, general relativity is required to describe the macroscopic structure of the universe, the macrocosmos. Thus, a final theory must provide a single framework capable of providing insights into the nature of space, time, all forces, and all matter ${ }^{12}$. The 1970s synthesis of the standard model, which is based on quantum mechanics and integrates three non-gravitational forces and their action on matter, marks the first approximation of a final theor $y^{11-13}$. However, the standard model incorporates some arbitrary parameters, and it leaves out gravitation, and thus general relativity ${ }^{11-13}$. Indeed, quantum mechanics and general relativity are mutually incompatible ${ }^{11-13}$. In this regard, string theory is our first plausible candidate for a final theory ${ }^{11-17}$. From the principle that everything at its most microscopic level consists of combinations of vibrating strings, superstring theory (or string theory) proclaims that the observable particle properties in the standard model are a reflection of the various ways in which a string can vibrate ${ }^{12}$. In particular, string theory is the only theory to incorporate anything like gravitation ${ }^{11-}$ ${ }^{13}$. Furthermore, it allows scientists to explore highenergy physics, including the origin of the universe $\mathrm{e}^{11-}$ 13 .

Unfortunately, string theory has not yet been successful and there is not yet a final theory ${ }^{11-17}$. 
Several situations taken together create an impasse for string theory to be a final theory for describing our universe and reflecting the cosmos. With courage gathered from our successful experience in applying Einstein's principle theory to develop the success/failure system hypothesis, we consider the cosmos. String theory is actually five distinct categories of theories, and this ensemble, called Mtheory, can generate thousands of string theories ${ }^{11,12,14}$. It is widely believed that these different string theories are different ways of solving the same underlying theory, which is perhaps the final theory we seek $^{11}$. No one has yet identified the exact equations of this underlying theory ${ }^{11,12,14}$.

Two concepts have been proposed to consider why string theory may apply to our universe: the multiverse and the anthropic principle ${ }^{11,12}$. The multiverse means that our universe is but one of an enormous number, say, the $500^{\text {th }}$ power of 10 , of separate and distinct universes ${ }^{11,12,14}$. One may counter that the concept of the multiverse is asking extravagant claims of an ultimate theory ${ }^{12}$. The anthropic principle states that the laws of nature in our universe must allow the existence of intelligent beings that can question the laws of nature ${ }^{11,12}$. It would be reasonable to say that our existence plays an important part in explaining why the universe is the way it is ${ }^{11,12}$. However, current research, which seeks a final theory, tends to make vague statements or prescribe values for the constants of nature in a range more or less favourable to life $e^{7,11,12}$. This means that the rationality of our existence in our universe in terms of laws of nature has been changed to a surrogate in practice: our universe allows life to form $^{12}$. A theoretical upheaval for the correct mathematical integration of quantum mechanics and general relativity may not address this impasse ${ }^{12,15,18}$. Neither is a sharp relief, such as the confirmation of supersymmetry through the discovery of superpartner particles in the origin of the universe ${ }^{11,12,17}$.

Einstein considered that understanding of logicalphilosophical arguments is important in advancing a unified theory ${ }^{6}$. Indeed, a final theory of the lifeladen universe may need to incorporate the success/failure system hypothesis (success/failure system) into its unifying framework. This is due to the sense of inevitability of applying the anthropic principle in the multiverse in final theor $y^{11,12}$. In addition to the anthropic principle in practice ${ }^{\mathrm{e} . \mathrm{g} .19}$, a universal biological law, the success/failure system exposes further the (life-laden) mesoscopic structure of the universe, the mesocosmos, to make it distinct from other universes without life. Now, we have a more complete set of laws of nature for building up the cosmos. A final theory of our universe may not be a duo of quantum mechanics and general relativity, the two main physical theories developed in the twentieth century, but a triad of quantum mechanics, general relativity and success/failure
ISSN 2455-6378

system. The theory accounts for everything by including three defined levels of the universe.

A final theory of our universe in its ultimate form is Einstein's principle theory ${ }^{4,6}$, which depicts the inner workings of the theory. Eventually, the cosmos will become a single logical system ${ }^{4}$. Scientists' collective ability will cause this discovery, which would be humanity's great victory. On that day of discovery, the cosmos, the ancient Greeks' and humanity's dreams, will be nakedly clear. The implications will be very rich. The cosmos is what Einstein wanted to know, so-called God's thoughts, as a pioneer in theoretical science with cosmic religious feeling ${ }^{6}$. Einstein considered the rest to be details $^{6}$. Thus, science will proceed in a systematic way as presently, with the partnering of empirical science and theoretical science.

\section{Conclusions}

We aimed to address the longstanding issue of universal laws in biology by applying Einstein's principle theory. The success/failure system hypothesis is, we believe, the only universal biological principle, and, as a highly constraining principle theory, reproducible and independent of the human factor. Considering the anthropic principle with respect to the multiverse in a final theory, the success/failure system hypothesis may need to be incorporated into a final theory of our universe. We propose that in the twenty-first century this simple effort may bring scientists to collectively realize a final theory of our universe and to grasp the cosmos far earlier than predicted. From our viewpoint, the reason a final theory of our universe is elusive is that scientists do not have a more complete set of laws of nature to work with. The success/failure system hypothesis, which reflects the mesoscopic structure of the universe, may resolve this issue. For ensuring this logical structure of the universe, we must next use Russell's analytic philosophy to examine critically the logic of the success/failure system.

\section{Acknowledgments}

The author would like to thank Anthony Abram for editing and proofreading this manuscript. I am grateful to Suzanna Rona for advice and feedback on the manuscript.

\section{References}

[1] Beatty, J. The Evolutionary Contingency Thesis. In: Concepts, Theories, and Rationality in the Biological Sciences, The Second PittsburghKonstanz Colloquium in the Philosophy of Science, University of Pittsburgh Press, Pittsburgh, 45-81, (1995). 
[2] Mayr, E. This is Biology: The Science of the Living World Harvard Univ. Press: 15, 62 (1997).

[3] Mayr, E. What Makes Biology Unique? Considerations on the Autonomy of a Scientific Discipline Cambridge Univ. Press: 26 (2004).

[4] Einstein, A. Ideas and Opinions Bonanza Books: 226, 293 (1954).

[5] Isaacson, W. Einstein: His Life and Universe Simon \& Schuster: 220 (2007).

[6] Calaprice, A. The Ultimate Quotable Einstein Princeton Univ. Press: 330, 368, 388, 396, 425 (2010).

[7] Bau, D. Y. The success/failure system hypothesis. IJASRM 3 (3): 30-34 (2018). http://ijasrm.com/wpcontent/uploads/2018/03/IJASRM_V3S3_496_3 0_34.pdf

[8] Stewart, I. Life's Other Secret: The New Mathematics of the Living World John Wiley \& Sons, New York (1998).

[9] Wilson, E. O. Naturalist Island Press, Washington (1994).

[10] Dyson, F. Infinite in All Directions Harper \& Row, New York (1988).
[11] Weinberg, S. Dreams of a Final Theory Vintage Books: 220, 229 (1992).

[12] Greene, B. The Elegant Universe: Superstrings, Hidden Dimensions, and the Quest for the Ultimate Theory W. W. Norton \& Company, New York (1999).

[13] Weinberg, S. Facing up: Science and Its Cultural Adversaries Harvard Univ. Press: 7, 173-174, 238-239 (2003).

[14] Hawking, S. \& Mlodinow, L. The Grand Design. Bantam Books, London (2010).

[15] Khrennikov, A. The present situation in quantum theory and its merging with general relativity. Found. Phys. 47: 1077-1099 (2017).

[16] Musser, G. What is spacetime? Nature 557: S3S6 (2018).

[17] Ananthaswamy, A. How the belief in beauty has triggered a crisis in physics. Nature 558: 186-187 (2018).

[18] Wu, Y. L. Unified field theory of basic forces and elementary particles with gravitational origin of gauge symmetry in hyper-spacetime. Sci. Bull. 62: 1109-1113 (2017).

[19] Meißner, U. G. Anthropic considerations in nuclear physics. Sci. Bull. 60: 43-54 (2015). 